\title{
An Introduction to the Auger Engineering Radio Array (AERA)
}

\author{
Amin Aminaei ${ }^{1}$ for the Pierre Auger Collaboration ${ }^{2,3}$
}

\begin{abstract}
The new generation of radio-telescope arrays with digital interferometry is able to measure electromagnetic pulses from ultra-high energy cosmic rays. Following the success of LOPES and CODALEMA, AERA is developed to detect and characterize the cosmic rays via the coherent broadband emission produced in the air showers. The results will provide a better understanding of the radio-emission mechanisms in air showers and will evaluate the capability of radio detection of cosmic rays for large-area hybrid observations.
\end{abstract}

ISKAF2010 Science Meeting - ISKAF2010

Assen, the Netherlands

June 10-14 2010

1 Institute for Mathematics, Astrophysics and Particle Physics, (IMAPP), Radboud Universiteit

Nijmegen, Huygens building, Department of Astrophysics, Faculty of Science, 6525 AJ, The Netherlands, Email: a.aminaei@science.ru.nl

2 Av. San Martín Norte 304 (5613) Malargüe, Prov. de Mendoza, Argentina

${ }^{3}$ A full author list and affiliations can be found at http://www.auger.org/archive/authors_2010_08.html 


\section{Introduction}

The Pierre Auger Observatory measures the highest energy cosmic rays. Cosmic rays have energies up to $10^{20} \mathrm{eV}$ and higher. While supernovae remnants are a likely source for the cosmic rays in the Galaxy, there is no preferred explanation for the acceleration of the ultra-high energy cosmic rays (UHECRs), which are believed to have extragalactic sources. The flux of cosmic rays decreases sharply when the energy increases. For instance, cosmic rays with energies of about $10^{19} \mathrm{eV}$ hit the Earth with a rate of 1 particle per square kilometer per year. To record a sufficient number of these events, the Pierre Auger Observatory has a large detection area in the western Argentina's Mendoza Province, with size of $3000 \mathrm{~km}^{2}$. The Pierre Auger Observatory is a hybrid air-shower detector, combining a surface detector (SD) and a fluorescence detector (FD). The SD detects the Cherenkov light emitted due to the interaction of the particle-shower front with the water inside SD tanks. The FD detects ultraviolet light emitted by nitrogen molecules that are excited by air showers in the Earth's atmosphere. A third way of cosmic-ray detection is based on geomagnetic separation of electrons and positrons of an extensive air shower (EAS) [1]. As a result, an EAS emits coherent radio pulses which can be measured by a radio-antenna array such as AERA. The combination of AERA with existing techniques can be used for the observation of a poorly understood part of the cosmic-ray spectrum, the galactic to extra-galactic transition region between $10^{17}$ and $10^{19} \mathrm{eV}$. Here we introduce the system characteristics of AERA and the preliminary results of prototype installations.

\section{The AERA site layout}

AERA is located near Malargüe in Argentina, and will cover $20 \mathrm{~km}^{2}$ of the Pierre Auger Observatory. The complete array will include 150 stations. The layout is illustrated in Fig. 1. Around the core, there will be 60 stations on a triangular grid with a pitch size of $250 \mathrm{~m}$. The outer region of AERA will have 72 stations with a distance between stations of $375 \mathrm{~m}$. The first 24 stations will be installed in September 2010. They act as the core stations and have a mutual distance of $150 \mathrm{~m}$. This core is about $5 \mathrm{~km}$ east of the Coihueco fluorescence detector. This location provides an excellent overlap for events which will be observed with both detection systems [2] but the duty cycle of fluorescence detectors is about $10 \%$ compared to $100 \%$ for AERA.

\section{The system characteristics of AERA}

A prototype AERA station is shown in Fig.2. The log-periodic dipole antenna (LPDA) and solar panels are visible while the electronics and digitizer are placed in the aluminum box. The AERA local stations are divided into subsystems which are briefly introduced. The LPDAs and analogue electronics (including Low Noise Amplifier and 
filter) are specifically designed to detect radio pulses from 30-80 MHz. The antennas are aligned with the magnetic north at the AERA site. Each antenna is sensitive to northsouth and east-west polarizations and has a low-gain and a high-gain channel for each

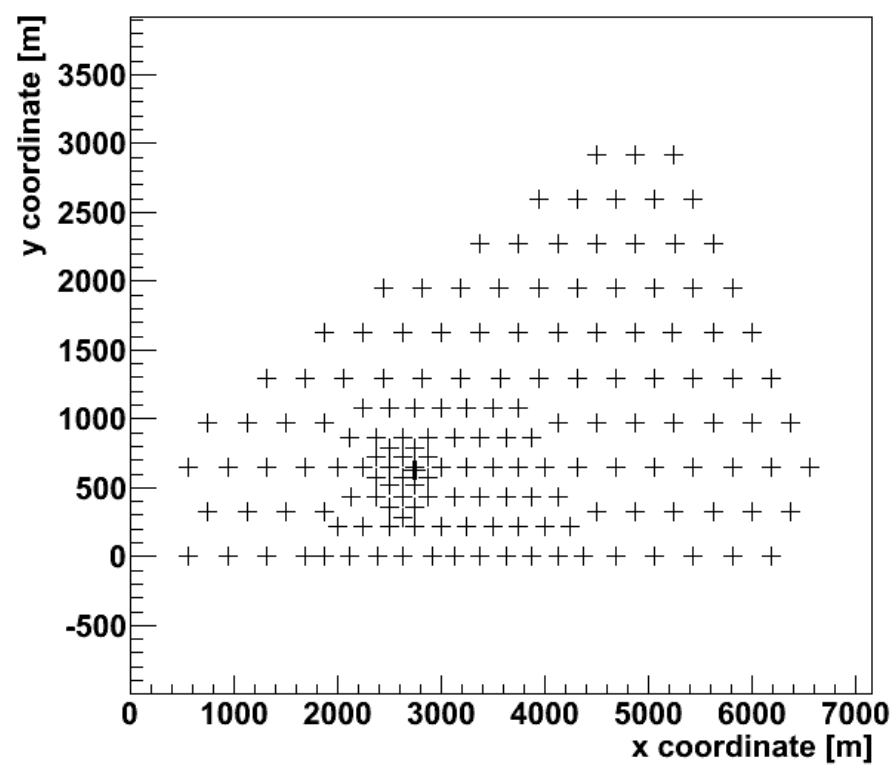

Fig. 1 The AERA site layout

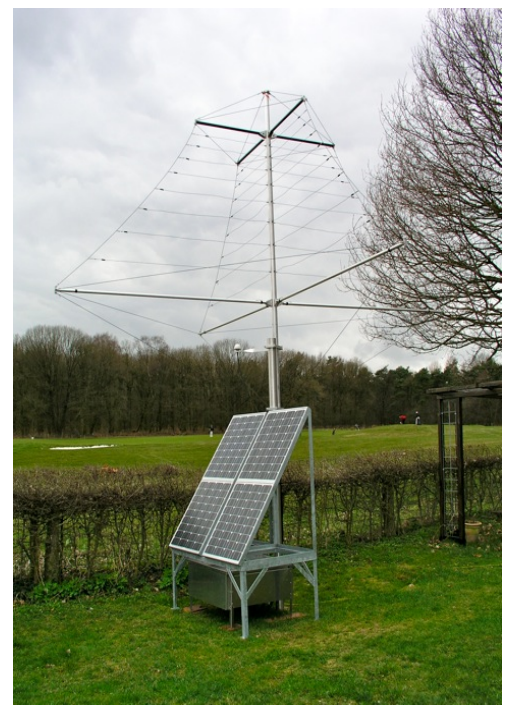

Fig.2 A prototype AERA station 


PoS $\quad$ PROCEEDINGS

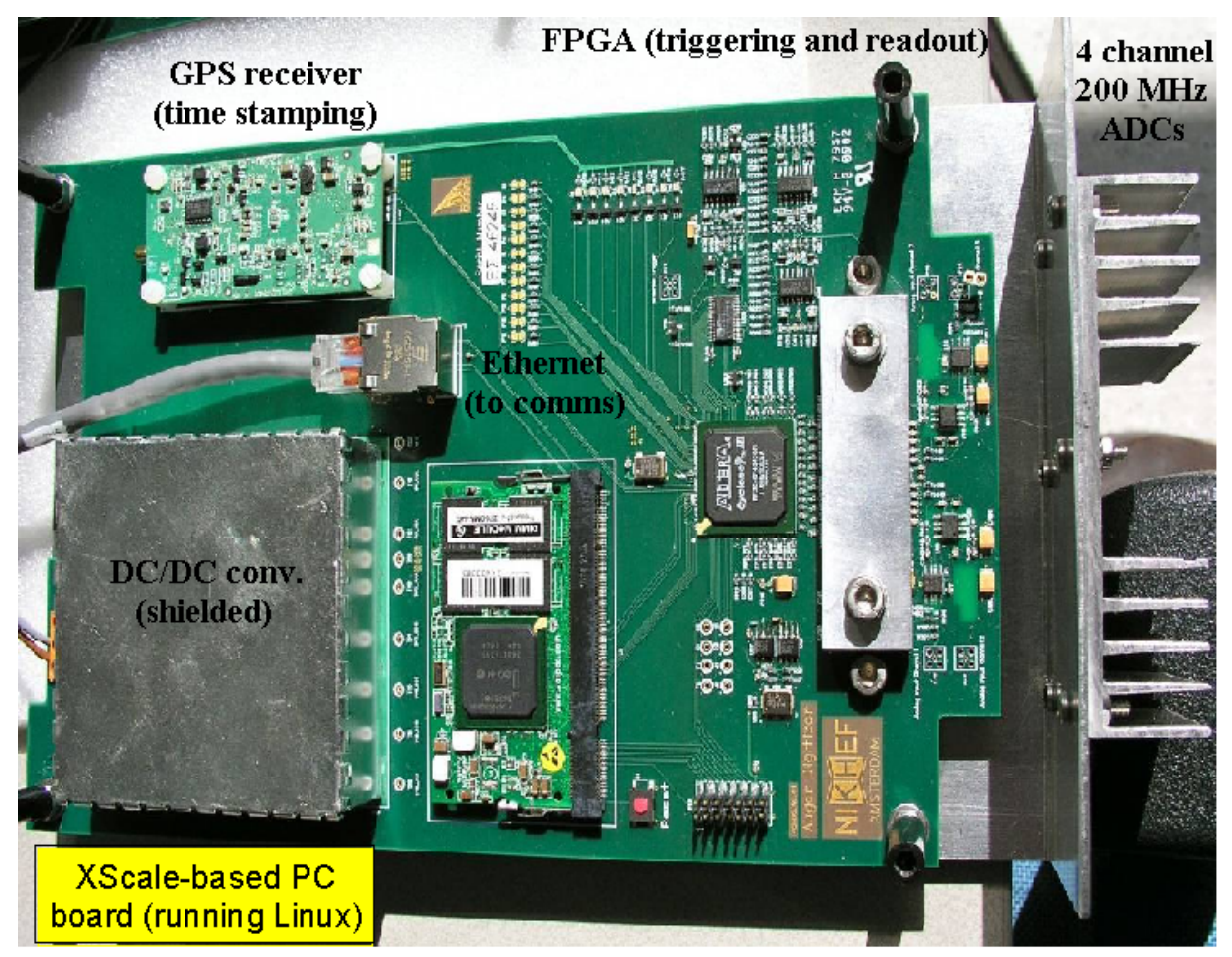

Fig.3 The electronics PC board at the local station

polarization. The filtered signal is digitized with 12-bit ADCs at sampling rate of 200 $\mathrm{MHz}$ and is stored in the local-station electronics. A Field-Programmable Gate Array (FPGA) which runs with Linux is used for managing the data storage and triggering (Fig.3). By triggering, the incoming streams of data are filtered for interesting events. A modified digitizer with 2 GByte RAM memory and sampling rate of $180 \mathrm{MHz}$ is currently being tested. RAM stores the data temporarily before they are filtered. Selected data are then collected by a central data acquisition (DAQ). The GPS antenna is used for time synchronization between stations.

For communication between local stations and the central DAQ, fiber optics are used for the initial phase. A high-speed, low-power wireless communication system is under development for subsequent phases. Using this wireless system, a data rate of $100-200$ kbps between stations will be possible.

A reference beacon [3] will be used for timing accuracy between stations (Fig.4). Using this beacon, a time accuracy of better than $1 \mathrm{~ns}$ is expected. 


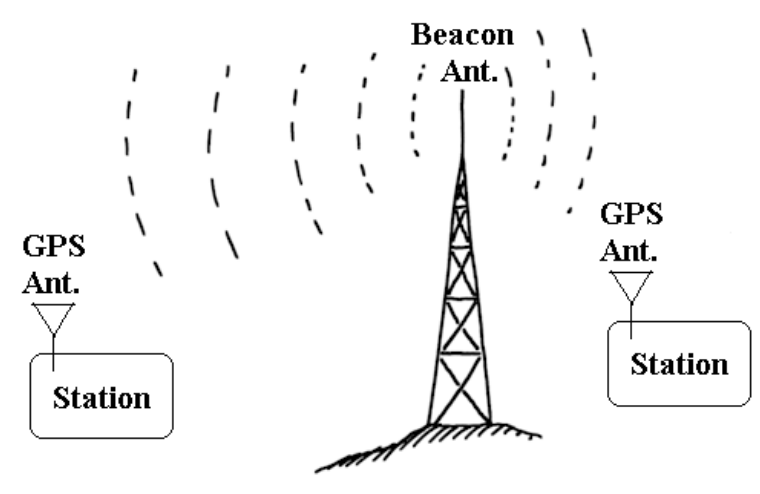

Fig.4 AERA stations are calibrated via a reference beacon

\section{Simulation and preliminary result}

The simulated AERA sensitivity to cosmic-ray radio pulses is depicted in Fig.5. For the radio pulses with energies of $10^{19.5} \mathrm{eV}$ the effective area is more than $75 \%$ of the total area $\left(20 \mathrm{~km}^{2}\right)$.
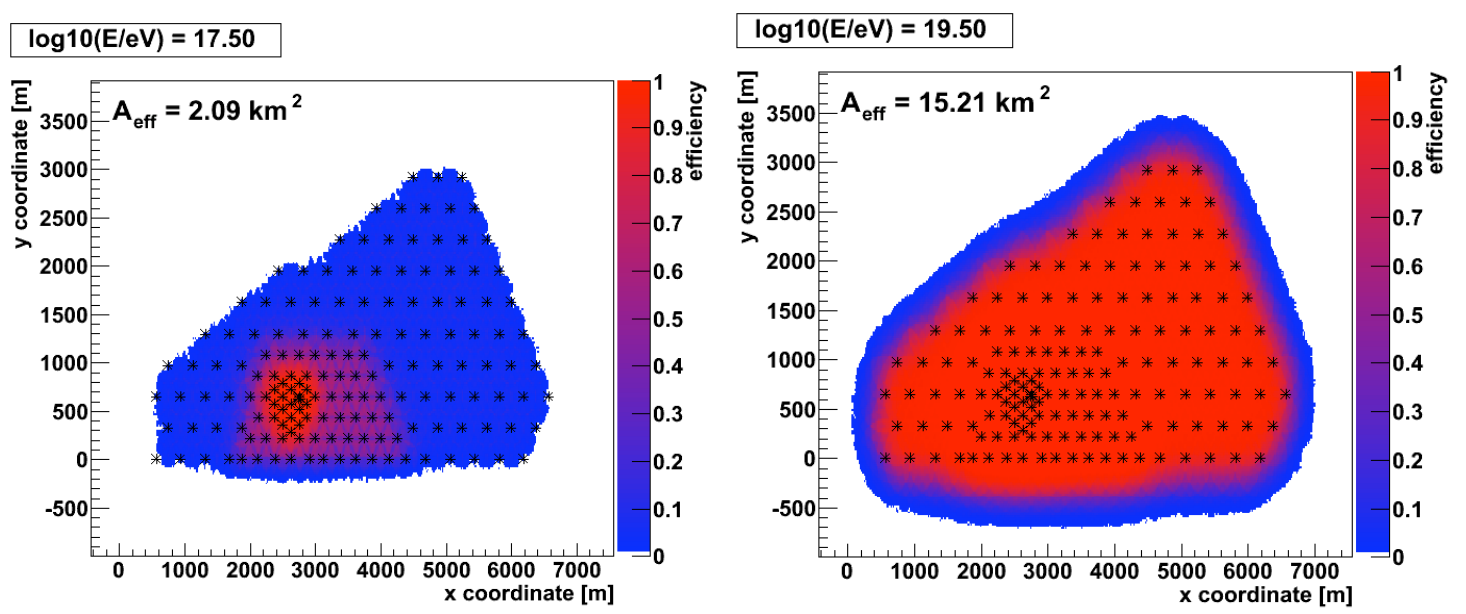

Fig.5 The simulated detector efficiency of AERA to radio pulses from cosmic-ray air showers of $10^{17} \mathrm{eV}$ and $10^{19.5} \mathrm{eV}$

Some result from prototype installations in Argentina (MAXIMA) is illustrated in Fig.6. It shows the variation of the background noise level vs. the local sidereal time caused by passage of the Galactic centre. The data are measured over one year and cover the frequency range between 50 to $55 \mathrm{MHz}$. 


\section{Summary}

To summarize, AERA is a step forward towards the large-scale radio detection of cosmic rays. This is particularly important to study the cosmic-ray parameters at the higher energies in the transition region between galactic and extragalactic sources.

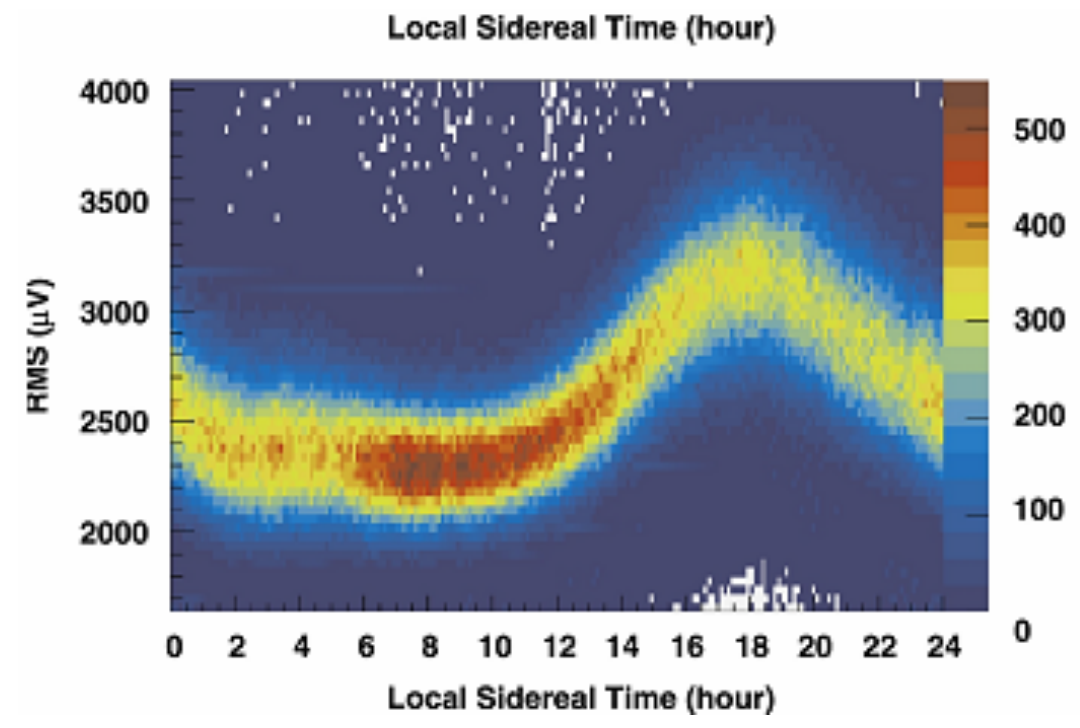

Fig.6 Result [4] from the initial prototype installations. The galactic noise measured between 50-55 MHz.

\section{References}

[1]F.D. Kahn and I. Lerche, Proc. Royal Soc. London A 289, p. 206, (1966)

[2] A. M. van den Berg et al., Proc. 31st Intl. Cosmic Ray Conf., arXiv:0908.4422.

[3]F. G. Schröder et al., Nucl. Inst. and Meth. A 615, 277 (2010)

[4] T. Huege et al., Nucl. Inst. and Meth. A 617, 484 (2010) 